\title{
Erratum to: Using a Crop Growth Model to Quantify Regional Biogas Potentials: an Example of the Model Region Biberach (South-West Germany)
}

\author{
Benjamin Mast • Wilhelm Claupein •
} Simone Graeff-Hönninger

Published online: 24 April 2014

(C) Springer Science+Business Media New York 2014

Erratum to: Bioenerg Res (2014)

DOI 10.1007/s12155-014-9442-8

The original version of this article unfortunately contained mistakes in Table 5. The correct version is presented below.

Table 5 Management scenarios and the associated sowing and harvest dates as well as vegetation period length [day] of maize and triticale

\begin{tabular}{|c|c|c|c|c|c|c|}
\hline \multirow[t]{2}{*}{ Management systems } & \multicolumn{2}{|l|}{ Maize } & \multicolumn{2}{|l|}{ Triticale } & \multirow{2}{*}{$\begin{array}{l}\text { Maize } \\
\text { Vegetation-period [day] }\end{array}$} & \multirow{2}{*}{$\begin{array}{l}\text { Triticale } \\
\text { Vegetation-period [day] }\end{array}$} \\
\hline & Sowing & Harvest & Sowing & Harvest & & \\
\hline Maize & 1 May & 30 Sept. & & & 153 & \\
\hline Triticale & & & 1 Oct. & 1 July & & 273 \\
\hline System 1 & 1 May & 30 Sept. & 1 Oct. & 30 April & 153 & 212 \\
\hline System 2 & 1 May & 14 Oct. & 15 Oct. & 30 April & 167 & 198 \\
\hline System 3 & 20 May & 14 Oct. & 15 Oct. & 19 May & 148 & 217 \\
\hline System 4 & 20 May & 30.Sept. & 1 Oct. & 19 May & 134 & 231 \\
\hline
\end{tabular}

The online version of the original article can be found at http://dx.doi.org/ $10.1007 / \mathrm{s} 12155-014-9442-8$.

B. Mast $(\bowtie) \cdot$ W. Claupein $\cdot$ S. Graeff-Hönninger

Institute of Crop Science, University of Hohenheim, Fruwirthstrasse

23, 70599 Stuttgart, Germany

e-mail: benjamin.mast@uni-hohenheim.de 